\title{
IRAK-M knockout promotes allergic airway inflammation, but not airway hyperresponsiveness, in house dust mite-induced experimental asthma model
}

\author{
Xudong Zhang ${ }^{1 \#}$, Mingqiang Zhang ${ }^{1 \#}$, Lun $\mathrm{Li}^{1}$, Wei Chen ${ }^{2}$, Wexun Zhou ${ }^{3}$, Jinming Gao ${ }^{1}$ \\ ${ }^{1}$ Departments of Pulmonary and Critical Care Medicine, Peking Union Medical College Hospital, Chinese Academy of Medical Sciences \& Peking \\ Union Medical College, Beijing, China; ${ }^{2}$ Departments of Cardiology, Peking Union Medical College Hospital, Chinese Academy of Medical \\ Sciences \& Peking Union Medical College, Beijing, China; ${ }^{3}$ Departments of Pathology, Peking Union Medical College Hospital, Chinese Academy \\ of Medical Sciences \& Peking Union Medical College, Beijing, China \\ Contributions: (I) Conception and design: J Gao; (II) Administrative support: J Gao, W Chen; (III) Provision of study materials or patients: X Zhang, \\ M Zhang; (IV) Collection and assembly of data: X Zhang, M Zhang, L Li, W Zhou; (V) Data analysis and interpretation: M Zhang, J Gao; (VI) \\ Manuscript writing: All authors; (VII) Final approval of manuscript: All authors. \\ "These authors contributed equally to this work. \\ Correspondence to: Jinming Gao, MD. Professor of Pulmonary Medicine, Department of Respiratory Diseases, Peking Union Medical College \\ Hospital, \#1 Shuaifuyuan, Dongcheng District, Beijing 100730, China. Email: gjinming@yahoo.com.
}

Background: IL-1 receptor associated-kinase (IRAK)-M, expressed by airway epithelium and macrophages, was shown to regulate acute and chronic airway inflammation exhibiting a biphasic response in an OVA-based animal model. House dust mite (HDM) is a common real-life aeroallergen highly relevant to asthma pathogenesis. The role of IRAK-M in HDM-induced asthma remains unknown. This study was aimed to investigate the effect of IRAK-M on allergic airway inflammation induced by HDM using IRAK-M knockout (KO) mice and the potential underlying mechanisms.

Methods: IRAK-M KO and wild-type (WT) mice were sensitized and challenged with HDM. The differences in airway inflammation were evaluated 24 hours after the last challenge between the two genotypes of mice using a number of cellular and molecular biological techniques. In vitro mechanistic investigation was also involved.

Results: Lung expression of IRAK-M was significantly upregulated by HDM in the WT mice. Compared with the WT controls, HDM-treated IRAK-M KO mice showed exacerbated infiltration of inflammatory cells, particularly Th2 cells, in the airways and mucus overproduction, higher epithelial mediators IL-25, IL33 and TSLP and Th2 cytokines in bronchoalveolar lavage (BAL) fluid. Lung IRAK-M KO macrophages expressed higher percentage of costimulatory molecules OX40L and CD 80 and exhibited enhanced antigen uptake. However, IRAK-M KO didn't impact the airway hyperreactivity (AHR) indirectly induced by HDM. Conclusions: The findings indicate that IRAK-M protects allergic airway inflammation, not AHR, by modifying activation and antigen uptake of lung macrophages following HDM stimulation. Optimal regulation of IRAK-M might indicate an intriguing therapeutic avenue for allergic airway inflammation.

Keywords: Allergic airway inflammation; house dust mite (HDM); IL-1 receptor associated-kinase-M (IRAK-M); T helper 2 immunity (Th2 immunity); macrophage

Submitted Jun 05, 2020. Accepted for publication Jan 07, 2021.

doi: $10.21037 /$ jtd-20-2133

View this article at: http://dx.doi.org/10.21037/jtd-20-2133

(C) Journal of Thoracic Disease. All rights reserved. 


\section{Introduction}

The airway epithelium constitutes the first barrier of host cellular defense against inhaled stimuli and regulates both innate and adaptive immune responses to inhaled aeroallergens (1). Allergic asthma is characterized by airway inflammation thought to be driven by an aberrant Th2 immunity, particularly eosinophils and CD4+ T helper 2 (Th2) cells $(2,3)$. Much evidence has outlined the key role of airway epithelium in determining the development and magnitude of type 2 responses during asthmatic process by sensing environmental aeroallergens via pattern recognition receptors (PRRs), in turn, activating antigen-presentation cells (APCs) and other innate immune cells, further leading to the release of the proinflammatory mediators, such as interleukin-33 (IL-33), IL-25, and thymic stromal lymphopoietin (TSLP), in a cell type-specific manner (1).

Toll-like receptors (TLRs) are a group of family member of PRRs belonging to TLR/interleukin (IL)-1 receptor superfamily and recognize house dust mite (HDM) $(1,4)$. HDM is the most common aeroallergen highly relevant to clinical expression of asthma and up to $85 \%$ of asthmatic individuals allergic to HDM (5). In mice development of asthma to HDM-specific responses depends on activation of airway epithelial TLR4 (6). Furthermore, Toll-interleukin-1 receptor (TIR)-8, a negative regulator of TLR4 signaling, was demonstrated to promote the development of HDMinduced innate and adaptive Th2 immunity (7).

Genome-wide analysis reveals that human chromosome $12 q 14.2$, containing the locus of IL-1 receptor-associated kinase (IRAK-M) gene, was genetically linked to asthma or asthma-related traits (8). Interestingly, genetic studies performed in childhood- and adult-onset individuals demonstrated the opposite effect of IRAK-M single nucleotide polymorphisms (SNPs) on asthma susceptibility $(9,10)$.

IRAK-M, expressed by airway epithelium and macrophages, negatively regulates TLRs signaling and inhibits downstream excessive inflammatory response $(9,11,12)$. However, accumulating evidence has shown that IRAK-M plays a distinct role, anti-inflammatory or proinflammatory, in regulation of inflammatory responses depending on various clinical scenarios and the kind of stimuli (13-16). The role of IRAK-M in modulating immunologic outcomes of asthma is also contextual. For example, IL-13-driven IRAK-M expression in airway epithelial cells inhibited TLR2 signaling that was associated with exacerbated airway inflammation $(12,17)$. By contrast, IRAK-M ablation attenuated dendritic cell (DC) activation, type 2 immunity, and airway pathology in an IL-33-induced allergic airway inflammation (18). We previously reported in asthma mouse models that IRAK-M knockout (KO) worsened acute airway inflammation to 24-day antigen exposure by enhancing Th2 and Th17 differentiation, but weakened chronic Th2 airway inflammation to 60 days of antigen challenge via maintaining DC-mediated Th1 activation $(10,19)$. It should be pointed out that we used an ovalbumin (OVA)-based animal model. However, there are several differences in phenotypes and mechanisms, existing between OVA-based model and models adopting a common environmental allergen. In addition, it has become evident that at least some of the underlying mechanisms that govern OVA-based models of airway inflammation differ from those induced by exposure to aeroallergens.

The OVA has relatively weak allergenicity and needs aluminum hydroxide as immunological adjuvant to induce a Th2 skewing model, thus has limitations in modeling allergic asthma $(20,21)$. A purified natural allergen HDM has been shown to offer a more relevant murine model of human allergic asthma (22).

In this current study, we adopted IRAK-M knockout (KO) mice to investigate the specific contribution of IRAK-M in HDM-induced allergic airway inflammation and airway hyperreactivity (AHR). We demonstrated a beneficial role for IRAK-M in attenuating Th2-dominated allergic airway inflammation through modulating macrophages overactivation and enhancement of antigen uptake by macrophage phagocytosis.

We present the following article in accordance with the ARRIVE Reporting Checklist (available at http://dx.doi. org/10.21037/jtd-20-2133).

\section{Methods}

\section{Mice}

IRAK-M KO mice (gifted by Dr. Nikolaos G. Frangogiannis, Albert Einstein College of Medicine) and WT mice (purchased from Experimental Animal Research Center, Beijing, China) on C57BL/6 background were maintained in a pathogen-free mouse facility at Peking Union Medical College Hospital Animal Care Center. Mice were used for experiments at age of 8-10 weeks.

Ethics approval for this study was obtained from the ethics committee of Peking Union Medical College Hospital for animal experimentation, in compliance with national or institutional guidelines for the care and use of 
animals (XHDW-2015-0047).

\section{Protocol for establishment of allergic asthma mouse model}

Protocol for sensitization and challenge was based on the previous published method with minor modification (23). Briefly, mice were injected intraperitoneally (i.p.) on days 0 , 3, 5 with HDM extract containing $10 \mu \mathrm{g}$ of Der p1 (Indoor Biotechnologies, VA, USA) in $200 \mu \mathrm{L}$ of sterile saline and then challenged intranasally (i.n.) with the same agent on days 10, 1214,16 , and 17. Control mice were received phosphate-buffered saline (PBS) during the sensitization and challenge phases. Mice were sacrificed 24 hours after the last challenge for airway reactivity measurement.

\section{Airway resistance testing}

According to previously reported method (24), specific airway resistance (sRaw) as an indicator of airway reactivity was measured at 24 hours after the last challenge using a double-flow plethysmograph (Pulmos-I. II. III, M.I.P.S., Osaka, Japan). Airway resistance was measured at baseline, followed by increasing concentrations of inhaled methacholine (MCh).

\section{Lung histology and immunobistochemistry}

Mice were sacrificed and the left lungs were removed, inflated to $25 \mathrm{cmH}_{2} \mathrm{O}$ with $10 \%$ formalin, fixed overnight, then embedded in paraffin, and sectioned at $4 \mu \mathrm{m}$ thickness as previously described (19). Lung sections were stained with hematoxylin \& eosin reagent. The severity of peribronchial inflammation was semiquantitatively graded in a blind manner according to published method (25).

Sections were stained with Alcian blue/periodic acid Schiff (PAS) to identify mucus-containing cells.

Masson trichrome staining was used to assess subepithelial fibrosis using an Image-Pro Plus analysis system (Media Cybernetics, Silver Spring, MD, USA) (25). Briefly, 5 digital photographs were taken at $200 \times$ magnification and the area of collagen deposition (AC) and the perimeter of basement membrane of bronchioles $(\mathrm{Pbm})$ were measured. Results were expressed as the area of AC per the perimeter of basement membrane of bronchioles (WAc/ $\mathrm{Pbm} \mu \mathrm{m}^{2} / \mu \mathrm{m}$ ). At least 5 bronchioles were randomly counted from each sample.

Immunohistochemistry (IHC) was used to detect protein expression of IRAK-M and $\alpha$-smooth muscle actin ( $\alpha$-SMA). Briefly, sections were incubated in sodium citrate buffer for 15 minutes at $80{ }^{\circ} \mathrm{C}$ for antigen retrieval, and then rinsed in $1.5 \% \mathrm{H}_{2} \mathrm{O}_{2}$ in methanol for 30 minutes to block endogenous peroxidase activity. After rinsing, sections were incubated with $5 \%$ rabbit serum for $30 \mathrm{~min}$ to block non-specific binding, and then incubated with primary antibodies overnight. After washing, sections were incubated with HRP conjugated secondary antibodies for $1 \mathrm{~h}$, developed with diaminobenzidine tetrahydrochloride (Dako, Hamburg, Germany). The primary antibodies used were goat anti-mouse IRAK-M (Abcam, Cambridge, UK) and goat anti mouse $\alpha$-SMA (Abcam, Cambridge, UK).

\section{Bronchoalveolar lavage (BAL) fluid collection and analysis}

Mice were euthanized 24 hours after the last challenge. BAL was performed following the established method of the lab (19). The lungs were injected 3 times with $0.7 \mathrm{~mL}$ ice-cold PBS (pH 7.4) and the fluid was recovered gently. The BAL fluid was spun at $1,500 \times \mathrm{g}$ for $5 \mathrm{~min}$ at $4{ }^{\circ} \mathrm{C}$, supernatant was collected and stored at $-70{ }^{\circ} \mathrm{C}$ until analyzed. Cell pellets were resuspended in $100 \mu \mathrm{L}$ of PBS. Total inflammatory cells were numbered on a hemocytometer in the presence of $0.4 \%$ trypan blue (Sigma, $\mathrm{MO})$. The determination of leukocyte differentials, B cells (CD3-B220+), CD4+T cells (CD3+CD4+B220-), and Th2 cells $(\mathrm{CD} 3+\mathrm{CD} 4+\mathrm{ST} 2+)$ was subjected to FACS according to our previously published methods $(10,19)$.

\section{ELISA analysis of cytokines in BAL fluid}

The levels of cytokines in BAL fluid were analyzed using commercially available specific OptEIA sandwich ELISA kits (eBioscience) as per manufacture's recommendations

\section{Serum concentrations of immunoglobulins (Igs) by ELISA}

Blood for measurement of serum Igs was collected 24 hours after the last challenge. Serum total $\mathrm{IgE}$ was measured using an ELISA kit (Abcam, Cambridge, UK) following the instructions of the manufacturer. An indirect ELISA method was used to assess the HDM-specific IgE and IgG1 levels in serum, OD values were read at $450 \mathrm{~nm}$ (26).

\section{Flow cytometry analysis}

Recovered cells were resuspended in $100 \mu \mathrm{L}$ of PBS. Gating for macrophages was set on the cells expressing 
both CD11c and Siglec-F. For detection of surface expression of costimulatory molecules on macrophages, cells were stained with fluorochrome (FITC, PE, PerCPCyanine 5.5, APC)-conjugated Abs (anti-mouse CD3, CD4, CD8, F4/80, CD11b, CD11c, CD40, CD80, CD83, CD86, OX40L) for 30 to 45 minutes at room temperature according to previously described method (19). After wash by PBS containing $0.1 \%$ sodium azide, cells were subjected on FACSCalibur (BD Biosciences). All fluorochromeconjugated Abs were purchased from eBioscience, BD Biosciences, or Biolegend. The control Abs were applied to the isotype samples.

\section{Extraction of $R N A$ and Semiquantitative real-time PCR (qRT-PCR)}

Total RNA was extracted from lung tissues using TRIzol reagent according to manufacturer's protocol. Reverse transcribed into complementary DNA (cDNA) using a commercial kit (TaKaRa). qRT-PCR was performed on the ABI 7500 Fast real time PCR System (Applied Biosystems) using SYBR Premix Ex Taq (TaKaRa). The relative gene expression levels were evaluated by the ratio to GAPDH mRNA. The specific primer pairs were follows: IRAK-M: forward 5'-GA AACATCTGTGGTACATGCCAGAA-3'; reverse 5'-ACTTTGCAGCCCGTTAGAACCTC-3'; GAPDH: forward 5'-TTGTCTCCTGCGACTTCAACA-3', reverse 5'-TGGTCCAGGGTTTCTTACTCC-3'.

\section{Phagocytosis assay}

Airway macrophages were isolated from BAL fluid as previously described $(10,19)$. Lung macrophages were isolated from lung tissue digested with collagenase type 1A and type IV bovine pancreatic DNase by centrifugation with Ficoll solution.

The FITC-dextran uptake assay was set up by incubating macrophages from the naïve mice of both genotypes with FITC-dextran at $4{ }^{\circ} \mathrm{C}$ for $30 \mathrm{~min}$ according to the published method (27). FACS analysis was performed. Median fluorescence intensity (MFI) was calculated.

\section{Statistical analyses}

Data are expressed as mean \pm SEM. Comparisons between multiple groups were done with analysis of variance (ANOVA), while comparisons between two groups were performed by Student's $t$-test (GraphPad Prism version 5.0, GraphPad, San Diego, CA). P value $<0.05$ was considered significant.

\section{Results}

\section{HDM challenge induced lung IRAK-M expression in the mouse model}

We previously showed that increased lung expression of IRAK-M in both acute and chronic mouse models induced by OVA $(10,19)$. It was tempting to examine whether lung IRAK-M expression was regulated by HDM in an allergic mouse model (Figure 1A). Consistently, significantly elevated expression of IRAK-M mRNA in the lungs was seen in HDM-treated mice compared to PBS-treated mice (Figure $1 B, \mathrm{P}<0.01$ ). IHC staining showed that lung epithelial IRAK-M was notably increased in HDM group compared with that in PBS control (Figure 1C).

\section{IRAK-M ablation worsened lung histopathology, not AHR, after HDM induction}

Given the inconsistency in the effect of IRAK-M on OVAinduced airway inflammation between acute and chronic models, we investigated whether IRAK-M deficiency had an impact on allergic airway inflammation induced by HDM in less than 3 weeks of exposure in a mouse model (28).

PBS treatment induced little airway inflammation in both IRAK-M KO and WT mice (Figure 2A, left panel). Compared with similarly-treated WT mice, HDMsensitized and challenged IRAK-M KO mice showed more severe allergic airway inflammation evidenced by thickened airway epithelium and more infiltration of inflammatory cells in the peribronchus and around vessels (Figure $2 A$, right panel). We semi-quantitatively scored the histopathological findings and found a significant increase in inflammation scores in IRAK-M KO mice compared with WT mice (Figure $2 B, \mathrm{P}<0.05$ ).

Consistent with worsened allergic inflammation in the airways, HDM-treated IRAK-M KO mice showed obvious mucus hypersecretion by goblet cells in the larger airways compared with their WT counterpart (Figure 2C,D).

AHR is one of key characteristics of asthma. We have previously shown the dual regulation of IRAK-M on airway reactivity in OVA-induced experimental asthma models $(10,19)$. We tested whether IRAK-M had an impact on AHR induced by HDM. HDM treatment increased the airway resistance in 


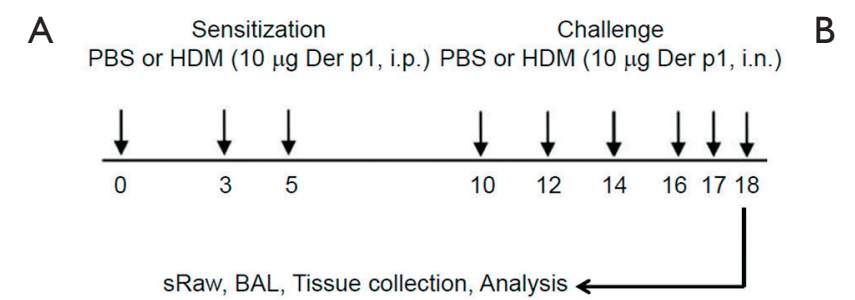

C

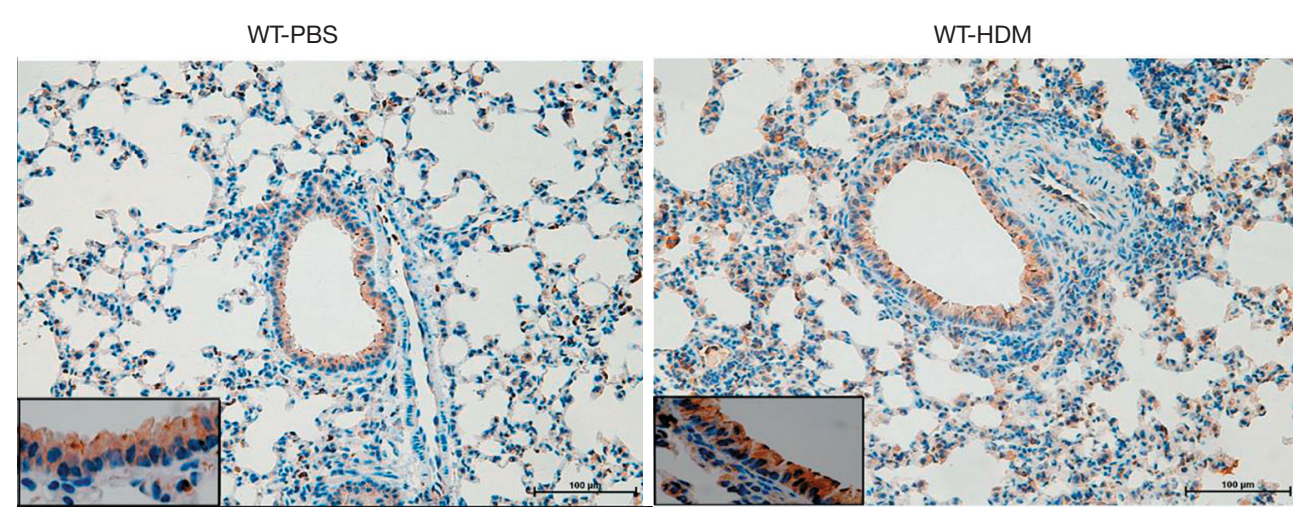

Figure $1 \mathrm{HDM}$ induced lung expression of IRAK-M in mice. (A) Schematic representation of the protocol for establishment of allergic asthma mouse model driven by HDM. (B) mRNA expression of IRAK-M in the lung of WT mice following PBS or HDM treatment. IRAK-M gene expression was normalized to GAPDH expression, $n=6$ mice in each group. * $\mathrm{P}<0.05$. (C) Representative immunohistochemical images of distribution of IRAK-M positive cells in the entire airways of mice treated with PBS (left panel) or HDM (right panel). HDM, house dust mite; IRAK-M, IL-1 receptor associated-kinase-M; WT, wild-type.

both WT and IRAK-M KO mice after the increasing doses of inhaled Mch, however, no significant difference was seen between the two groups of mice $(\mathrm{P}>0.05$ for all time points, Figure 2E).

\section{IRAK-M KO exacerbated HDM-induced Th2 immune response in the airways}

To determine whether IRAK-M depletion affected the HDM-induced infiltration of inflammatory cells into the airways, we estimated the cell subpopulations in BAL fluids following allergen sensitization and challenge. Following HDM challenge, the number of total inflammatory cells, macrophages, and neutrophils in BAL fluids was similarly increased between the both genotypes of mice, but there was significantly higher number of eosinophils and lymphocytes in the airways in HDM-sensitized and -challenged IRAK-M KO than in similarly treated-WT mice (Figure 3A).

Using flow cytometry analysis of different cell groups in BAL fluid, we found that percentages of Th2 cells, B cells, not CD4+T cells, were significantly higher in IRAK-M KO mice than those in WT mice (Figure 3B).

\section{Effect of IRAK-M deficiency on cytokines production in response to $H D M$}

Upon aeroallergen challenge, activated airway epithelial cells release alarming mediators such as IL-25, IL33, and TSLP which control downstream Th2 cellmediated immunity (1). We observed significantly elevated concentrations of IL-25, IL-33, and TSLP in BAL fluids from HDM-treated IRAK-M KO mice compared to those from similarly-treated WT mice (Figure 4A).

We next examined the effect of IRAK-M KO on cytokine production in the airways. After HDM challenge, the levels of Th2 cytokines such as IL-4, IL-5, and IL-13 in BAL fluids were significantly higher in IRAK-M KO mice than those in WT mice. There were markedly increased concentrations of Th1 cytokine IFN $\gamma$ and Th17 cytokine IL17A in BAL fluid from IRAK-M KO mice than those from WT mice. There were similar concentrations of Tregassociated cytokines IL-10 and TGF- $\beta$ between WT and IRAK-M KO mice (Figure 4B). 


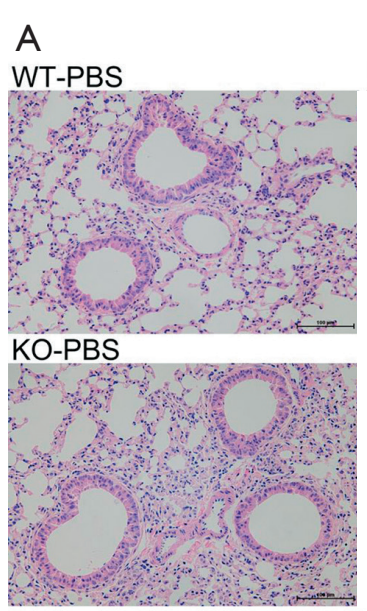

B

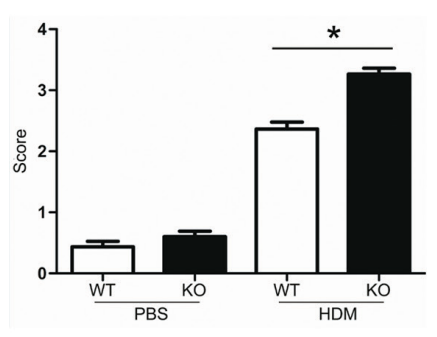

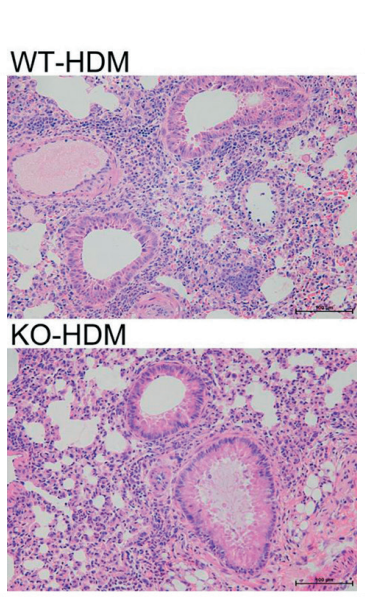

D

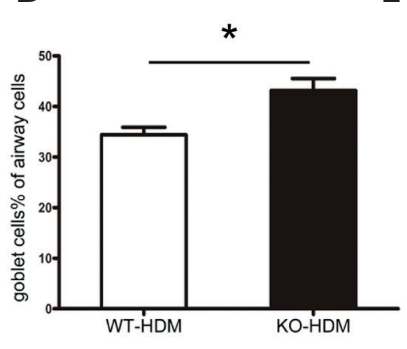

C

WT-PBS

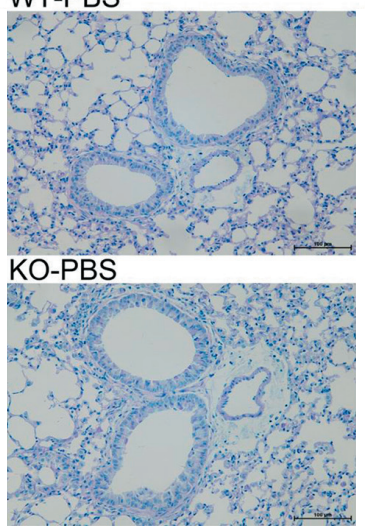

WT-HDM

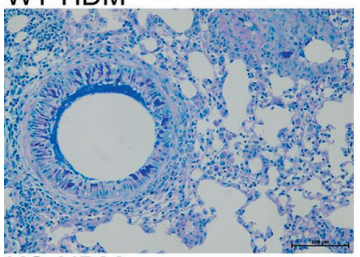

KO-HDM

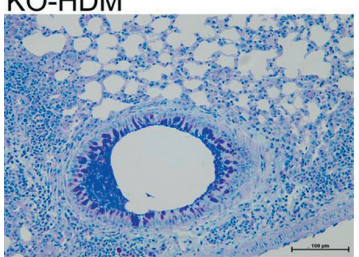

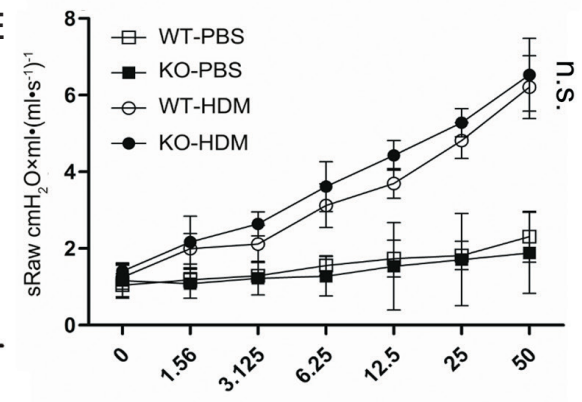

Methacholine $\mathrm{mg} / \mathrm{ml}$

Figure 2 IRAK-M KO worsened airway inflammation, not AHR, in mice after HDM induction. (A) Representative photomicrographs of HE-stained lung tissues in mice exposed to PBS or HDM. (B) Semiquantitative scorings of airway inflammation for PBS- or HDM-treated WT and IRAK-M KO mice. $\mathrm{n}=6$ mice in each group; ${ }^{*} \mathrm{P}<0.05$. (C) Representative photomicrographs of periodic acid-Schiff-stained lung tissues from mice exposed to PBS or HDM. (D) Histopathological evaluation of mucus-staining goblet cells, $\mathrm{n}=6$ animals per group, *, $\mathrm{P}<0.05$. (E) HDM-sensitized and challenged IRAK-M KO mice showed comparable AHR reflected by sRaw values in response to increasing doses of inhaled Mch compared to similarly treated-WT mice, n=6 animals in each group. n.s., not significant; HDM, house dust mite; IRAK-M, IL-1 receptor associated-kinase-M; WT, wild-type; KO, knockout.

\section{IRAK-M loss increased serum Ig levels}

Allergen-specific $\operatorname{IgE}$ is a key player in the pathophysiology of allergic asthma (29). We measured the levels of total $\mathrm{IgE}, \mathrm{HDM}$-specific IgE, and HDM-specific IgG1 in serum and found significantly increased serum levels of total $\mathrm{IgE}, \mathrm{HDM}$-specific IgE and IgG1 in IRAK-M KO mice compared with the WT mice (Figure 5).

\section{IRAK-M deficiency influenced expression of costimulatory molecules on macrophage after HDM induction}

Naïve T cells differentiation is tightly controlled by APCs, mainly including DCs and macrophages, via expressing costimulatory molecules (1). We first checked the expression of costimulatory molecules by DCs isolated from BAL fluids. However, there were no differences in expression of costimulatory molecules by DCs between IRAK-M KO and WT mice after HDM challenge (data not shown).

Macrophages, highly expressing IRAK-M, also affect differentiation of $\mathrm{T}$ cells by providing costimulatory molecules in an OVA-induced asthma model (19). In accordance with this notion, we observed that HDM treatment significantly increased expression of OX40L and CD80 by BAL macrophages from IRAK-M KO mice compared to those for WT mice (Figure 6).

\section{Effect of IRAK-M loss on antigen uptake by macrophage}

Macrophage phagocytosis, an important event in keeping local airway mucosal immune homeostasis, represents 

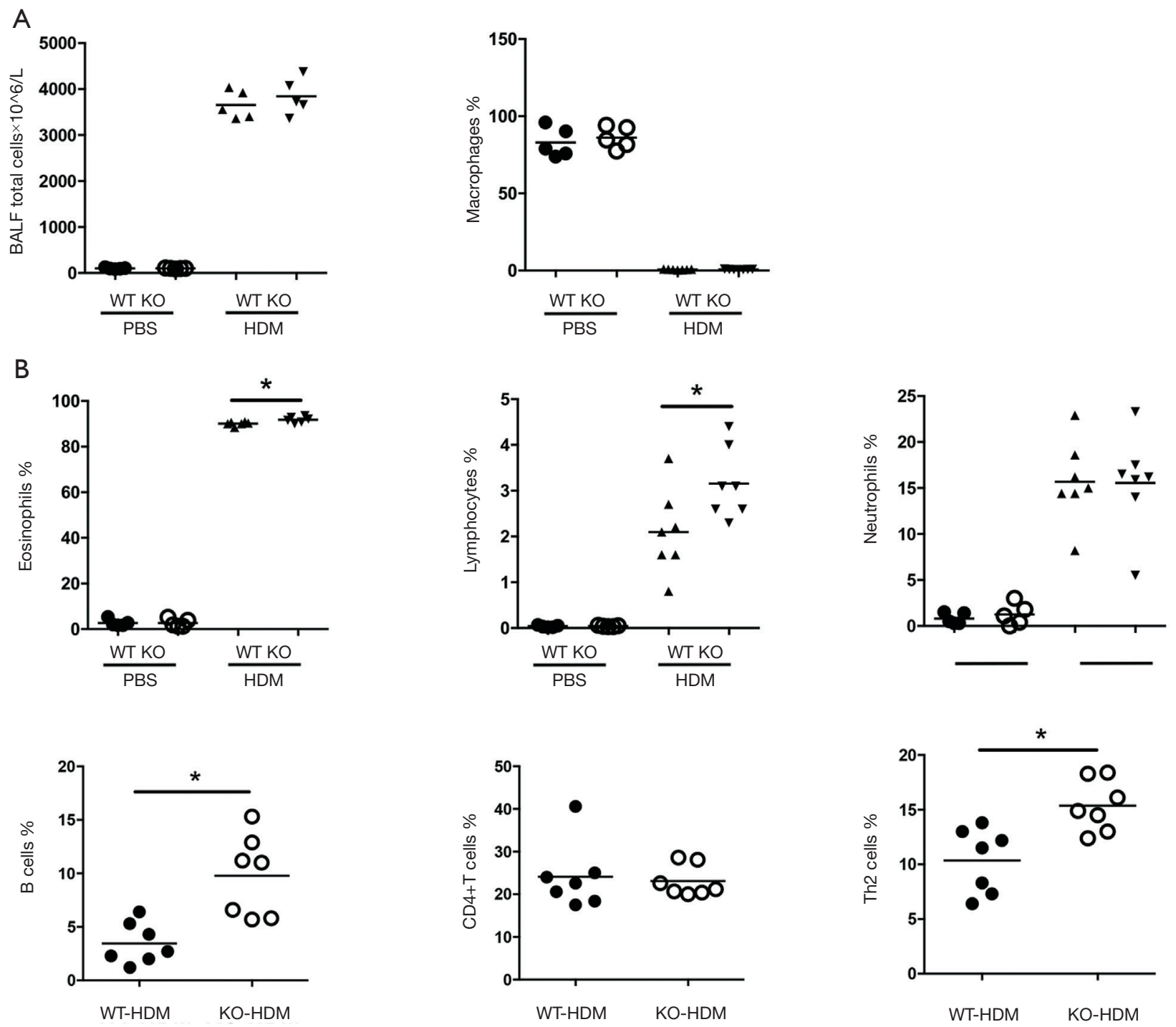

Figure 3 Assessment of infiltration of inflammatory cells in the airways. (A) Total inflammatory cells and differential subpopulations in BAL fluid. *, $\mathrm{P}<0.05$. (B) Summary plots of flow cytometry analysis for percentage of B, CD4+T, and Th2 cells in BAL fluids of WT or IRAK-M KO mice following HDM stimulation. *, $\mathrm{P}<0.05$. HDM, house dust mite; IRAK-M, IL-1 receptor associated-kinase-M; WT, wild-type; $\mathrm{KO}$, knockout; BAL, bronchoalveolar lavage.

an important process involved in antigen uptake (27). Dysfunction of this process may be contributing to maladaptive immunity $(30,31)$. Particles phagocytosis by airway macrophages was shown to be enhanced in mild asthma patients (32). Consistently, we found that IRAK-M $\mathrm{KO}$ macrophages isolated from BAL fluids and lungs demonstrated significantly enhanced capacity of antigen uptake by macrophage phagocytosis compared to WT macrophages (Figure 7).

\section{Effect of IRAK-M loss on airway subepithelial structure}

Increased collagen deposition and smooth muscle cells are the typical features of asthma pathology (33). We found that HDM treatment significantly increased collagen deposition around the airways in both WT and IRAK-M KO mice, but no significant difference was observed between the two groups of mice (Figure $8 A$ ). Of note, IHC staining showed that $\alpha$-SMA positive area per the perimeter of basement membrane of bronchioles was significantly increased in 
A

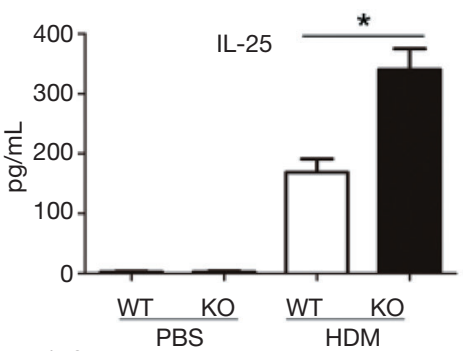

B

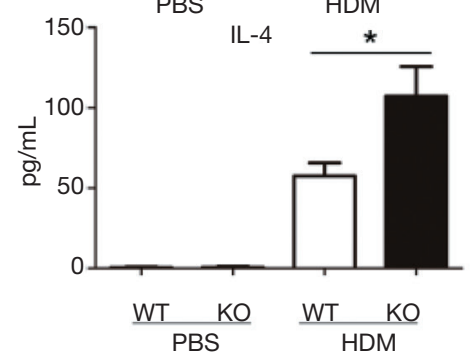

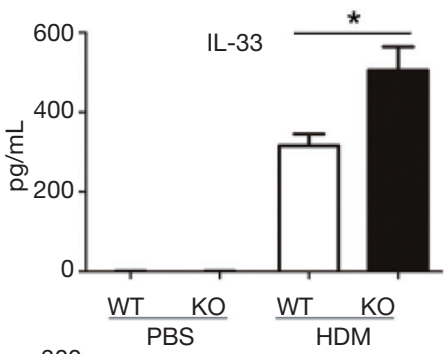

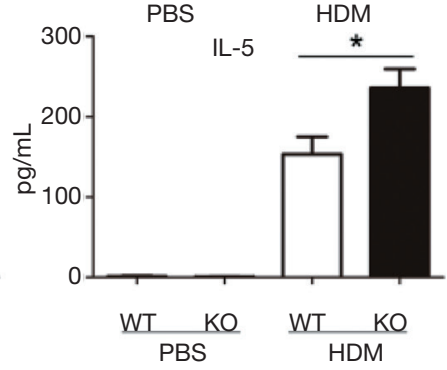

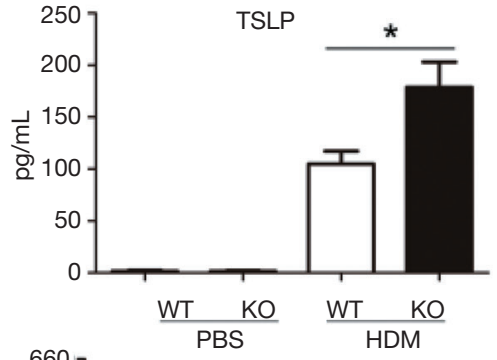

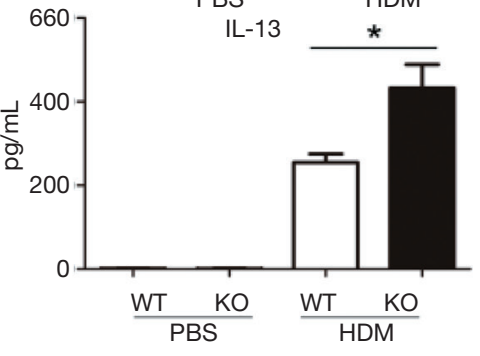

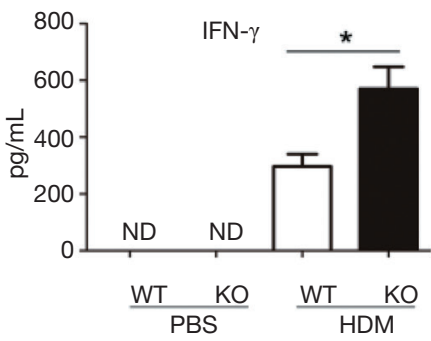
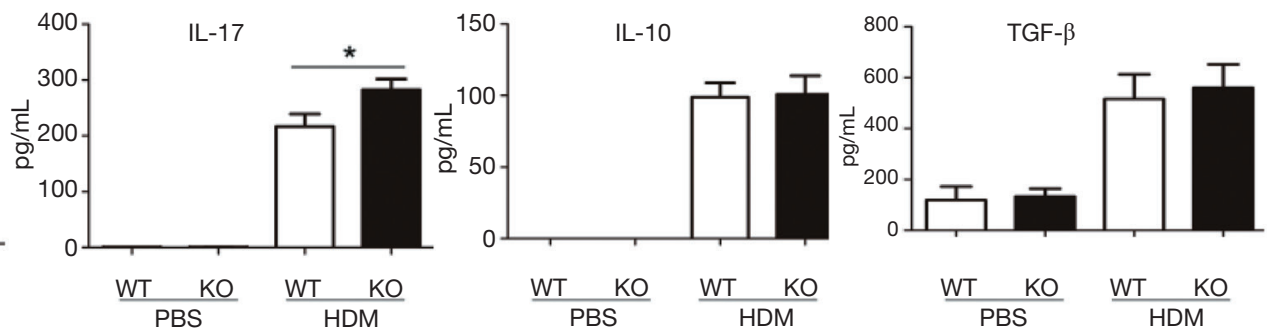

Figure 4 IRAK-M deficiency increased cytokine production in the airways after HDM treatment. (A) ELISA analysis for levels of airway epithelial cytokines IL-33, IL-25, and TSLP in BAL fluids, $n=5-10$ animals in each group, ${ }^{*}, \mathrm{P}<0.05$. (B) ELISA analysis for levels of various proinflammatory cytokines in BAL fluids from WT or IRAK-M KO mice following HDM stimulation, $\mathrm{n}=5-10$ animals in each group, *, $\mathrm{P}<0.05$. TSLP, thymic stromal lymphopoietin; HDM, house dust mite; IRAK-M, IL-1 receptor associated-kinase-M; WT, wild-type; KO, knockout; BAL, bronchoalveolar lavage.

IRAK-M KO mice than that in WT mice after HDM treatment (Figure 8B).

\section{Discussion}

The airway epithelial cells express a full complement of TLRs that recognize the environmental allergens such as HDM and regulate allergic airway inflammation (34), thus asthma is thought to be an airway epithelium disorder. We previously demonstrated a distinct effect of airway epithelial IRAK-M, a negative regulator of TLRs, on OVA-induced airway inflammation in animal model depending on the duration of challenge and disease stage $(10,19)$.

The OVA model uses aluminum hydroxide as an adjuvant for Th2-skewing immunity, thus it has drawbacks in studying innate immunity driving allergic airway inflammation. HDM contains immunogenic properties and is widely used to replicate allergic asthma mouse model (20). Using an HDMsensitized and -challenged protocol, IRAK-M KO mice showed: (I) a significant increase in the severity of allergic airway inflammation as evidenced by more inflammatory cells (particularly eosinophils and Th2 cells) in the airways, worsened airway pathology, and mucus overproduction; (II) more secretion of proinflammatory cytokines (including epithelial-release proinflammatory mediators and Th2 cytokines); (III) higher levels of serum IgE, HDM-specific IgE and IgG1; (IV) overactivation of macrophages exampled by higher expression of costimulatory molecules and in vitro enhanced antigen uptake shown by macrophage phagocytosis. Consistent with our previous observations in acute asthma model induced by OVA, IRAK-M may have an inhibitory role in infiltration of inflammatory cells into the airways, particularly Th2 cells, associated with allergic immune response to HDM in an experimental asthmatic 

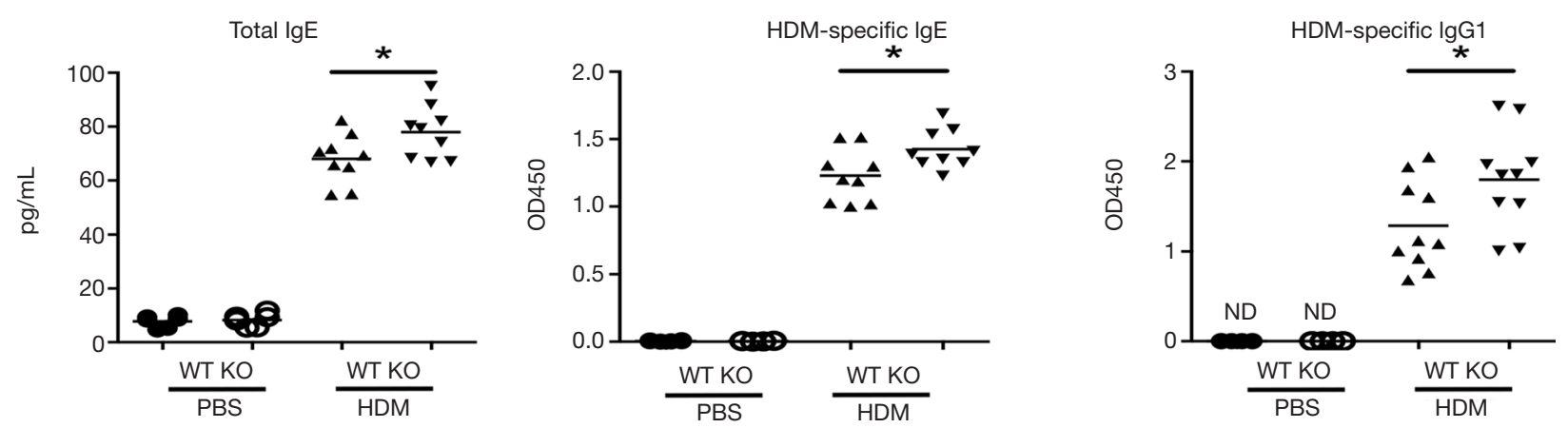

Figure 5 Elevation of serum levels of Igs in HDM-treated IRAK-M KO mice. ELISA analysis for serum concentration of total IgE, HDMspecific IgE and IgG1. *, P<0.05. HDM, house dust mite; IRAK-M, IL-1 receptor associated-kinase-M; KO, knockout.

A

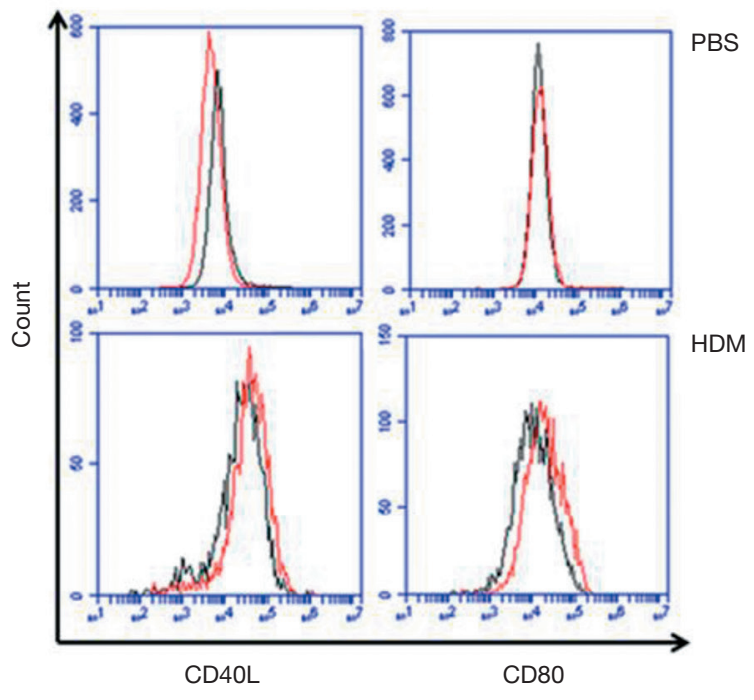

B
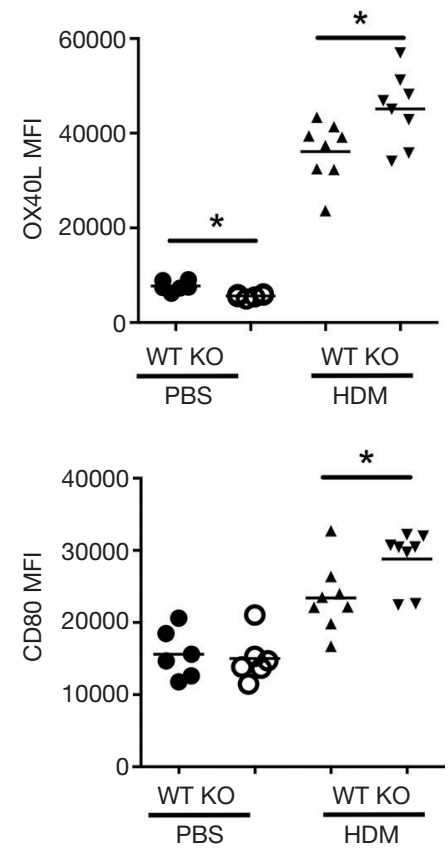

Figure 6 Induction of the expression of co-stimulatory molecules on macrophages following HDM treatment. Representative images (A) and summary plots of flow cytometry analysis of co-stimulatory molecules OX40L and CD80 on lung macrophages (B) from WT or IRAK-M KO mice after HDM or PBS treatment. *, P<0.05. HDM, house dust mite; IRAK-M, IL-1 receptor associated-kinase-M; W'T, wild-type; KO, knockout.

animal model.

Intracellular IRAK-M negatively regulates the TLR/ $N F-\kappa B$ signaling pathway to block inflammatory cascade and stabilize the airway mucosal immune system (11). For instance, IRAK-M KO increased infiltration of inflammatory cells and elevated expression of cytokines in an experimental mouse model of acute myocardial infarction (35). IRAK-M KO mice had a substantial increase in mortality after pulmonary influenza infection (36). IRAK-M deficiency promoted the development of earlyonset diabetes in mice (14).

Allergic asthma is often considered to be a disease driven by Th2-type responses to inhaled antigens (2). IRAK-M has been shown to play an important role in Th2 cell-mediated immunity and IL-33-induced allergic airway inflammation (18). The production of Th2 cytokines is 

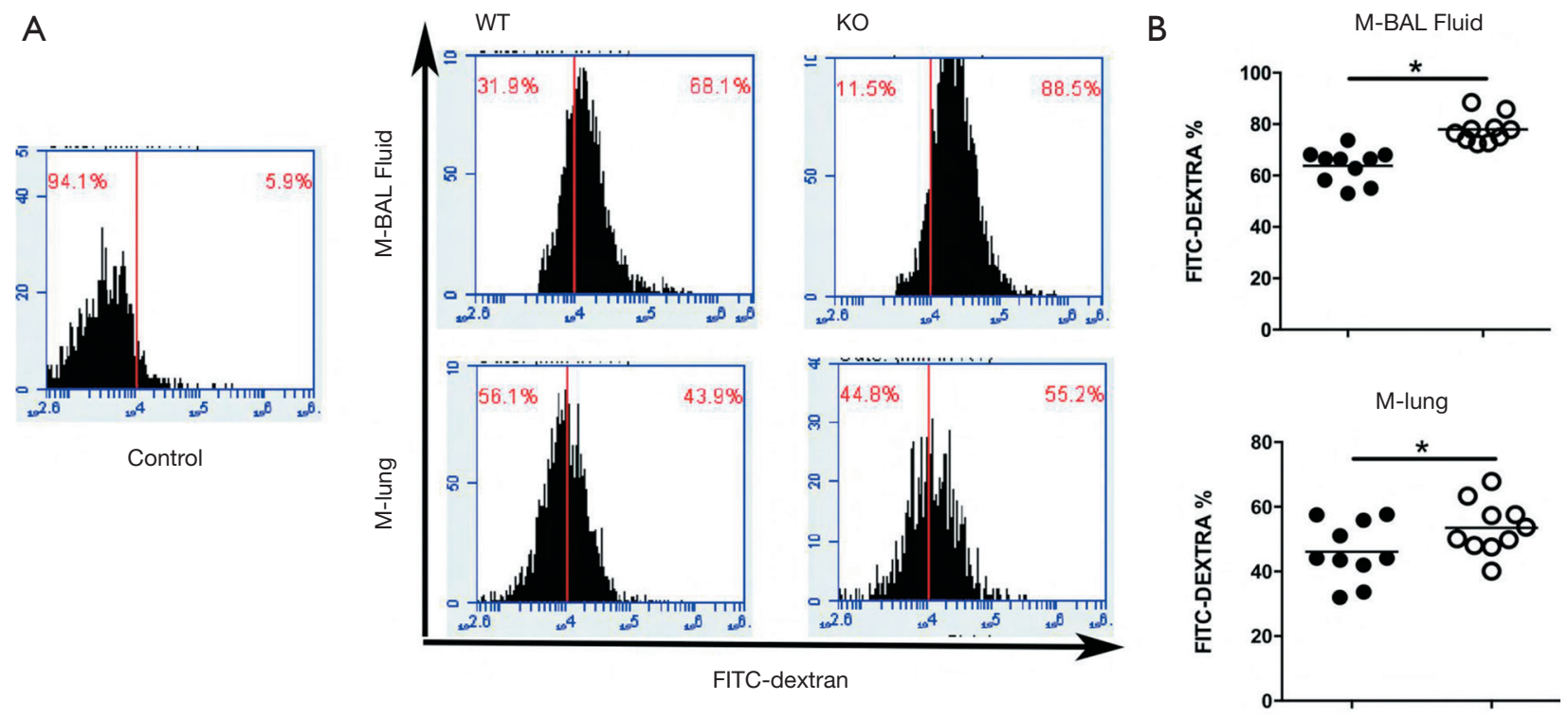

WT

$\mathrm{KO}$

Figure 7 IRAK-M knockout enhanced antigen uptake by macrophage phagocytosis. FACS analysis was performed and MFI was calculated. (A) Representative flow cytometric histograms of phagocytosis experiment of macrophages from BAL fluids (M-BAL fluid) and lungs (M-lung). (B) Summary plot of MFI for comparison of phagocytosis capacity by macrophages isolated from IRAK-M KO and WT mice. *, $\mathrm{P}<0.05$. HDM, house dust mite; IRAK-M, IL-1 receptor associated-kinase-M; WT, wild-type; KO, knockout; MFI, median fluorescence intensity; BAL, bronchoalveolar lavage.

regulated by upstream alarming molecules such as IL25, IL-33, and TSLP, which are released from airway epithelium upon activation of TLRs by inhaled allergens (1). We observed an increased IRAK-M immunoreactivity in airway epithelial cells after HDM challenge, and IRAK-M ablation led to significant elevation of BAL IL-25, IL33, and TSLP. Furthermore, BAL Th2 cytokines such as IL-4, IL-5, and IL-13 were higher in IRAK-M KO mice treated with HDM. These data suggest that IRAK-M might modulate expression of abnormal Th2 response in allergic airways via epithelium-derived mediators. We also demonstrated that HDM significantly increased serum levels of total IgE, HDM-specific IgE and IgG1 in IRAK-M $\mathrm{KO}$ mice. These might be due to higher expression of IL-4 in the airways because IL- 4 can promote $\operatorname{IgE}$ synthesis through induction of Th2 cell polarization (37). In this study using HDM-treated mouse model, IRAK-M KO mice not only demonstrated the significantly higher levels of BAL IL-4 (Figure 5B), but also significant elevation of IL-4 mRNA in the lungs (data not shown). Collectively, these data indicate the importance of IL-4 in IgE production.

The data presented in HDM-induced model showing higher concentrations of BAL Th1 cytokine IFN $\gamma$ and
Th17 cytokine IL17A in IRAK-M KO mice were consistent with our observations in acute and chronic asthma models induced by OVA, indicating the regulatory role of IRAK-M in T cells differentiation into Th1 or Th17 (38).

Airway epithelial innate molecules are instructive to APCs in the induction of Th2 cell differentiation through the differential expression of costimulatory molecules such as CD80, OX40L. Contrary to our findings in the OVA-induced acute asthma model (19), IRAK-M didn't influence expression of costimulatory molecules by airway DCs, the potent APCs, after HDM challenge. IRAK-M is predominantly expressed in macrophages and an inhibitor of macrophage function (11). In this present study, IRAK-M $\mathrm{KO}$ macrophages from the airways and lungs showed higher expression of costimulatory molecules OX40L and CD80 after HDM treatment. Furthermore, lung macrophages have demonstrated to be proinflammatory in mediating asthmatic airway inflammation (39). IRAK-M KO macrophages had higher levels of proinflammatory cytokines and greater costimulatory molecule expression in a septic mouse model (13). We and others have demonstrated that macrophages have the capability to regulate the pulmonary response to inhaled allergen including higher 


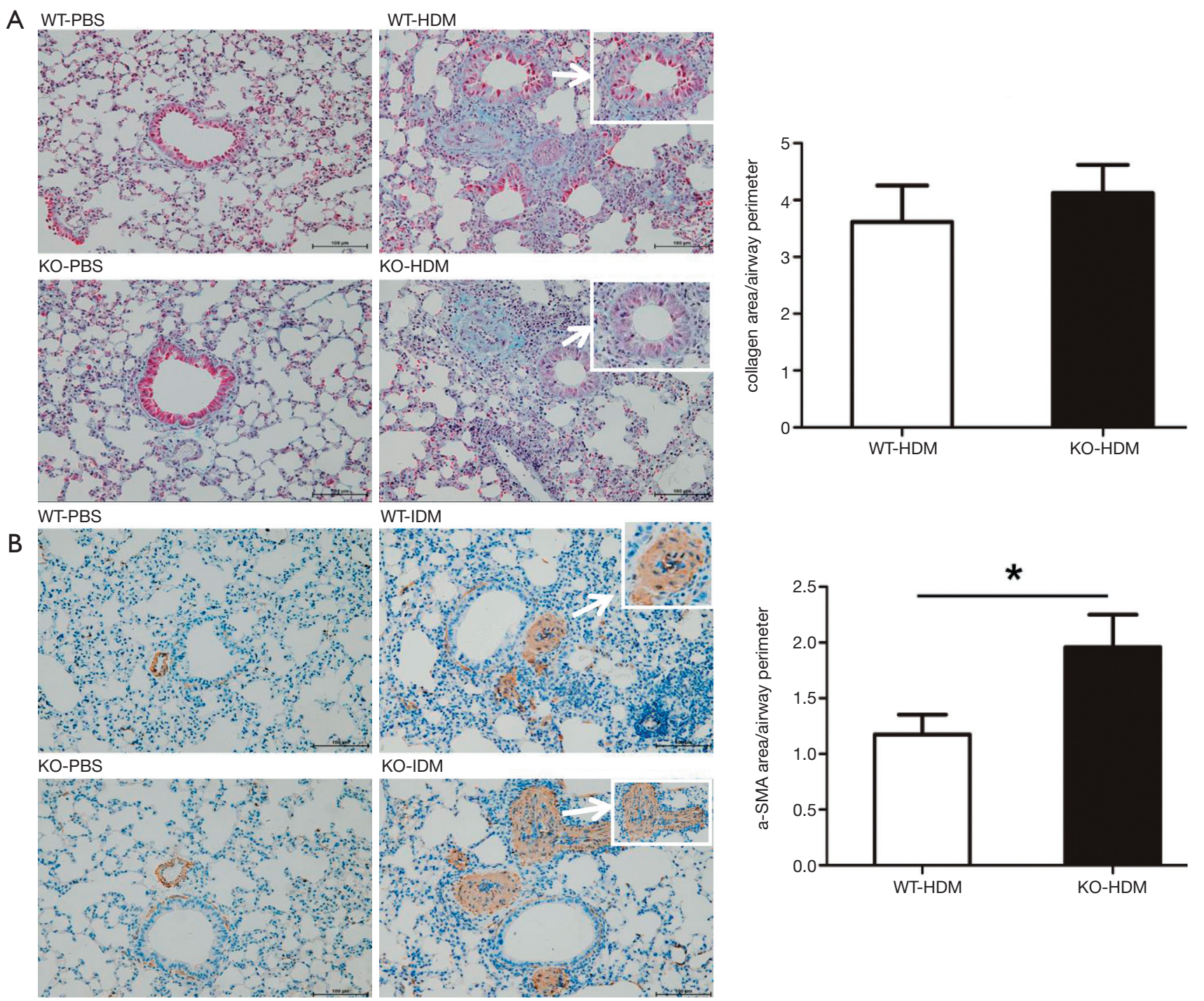

Figure 8 Airway subepithelial changes after HDM induction. (A) Representative images of Masson trichrome-stained lung tissue (left panel); Similar deposit of collagen around the airways between HDM-treated IRAK-M KO mice and WT mice (right panel), n=6 mice per group from two independent experiments, positive staining for collagen was shown as blue color. (B) Representative photomicrographs of immunohistochemical staining for a-SMA in the lungs (left panel); semi-quantitative evaluation of the difference in a-SMA area in the lungs from IRAK-M KO mice and WT mice after HDM induction (right panel), n=10 mice per group, positive staining for a-SMA was shown as brown color. *, P<0.05. HDM, house dust mite; IRAK-M, IL-1 receptor associated-kinase-M; WT, wild-type; KO, knockout.

expression of costimulatory molecules and production of cytokines during asthmatic process (19,39-42).

Because of abundant phagocytes, airway macrophages are the first immune cell to encounter inhaled allergens which are critical for maintaining airway mucosal hemostasis. On the other hand, macrophage phagocytosis has been shown to be dysfunctional and proinflammatory in asthma (39). In this study, we showed that HDM-treated IRAK-M KO mice had an enhanced antigen uptake shown by macrophage phagocytosis, leading to greater expression of costimulatory molecules OX40L and CD 80 by pulmonary macrophages, further promoting T cells skewing to Th2 type. Indeed, a previous study has shown that activation of lung macrophages drives Th2 immune response and also elaborates IL-17 release in an OVA-induced experimental animal model (38).

AHR is one of the primary features of allergic airway 
diseases. Contrary to the OVA-induced asthma model, IRAK-M didn't show the effect on airway reactivity in this HDM-induced model of allergic asthma. Previous studies showed that TGF- $\beta 1$ regulated AHR in an OVAbased model (43-45), not necessarily for the development of airway reactivity in HDM-induced animal model (46). AHR has been reported to be dissociated from airway inflammation in patients with chronic asthma (47). The lack of effect might reflect that AHR in asthma results from multiple mechanisms and is not closely related to airway inflammation $(47,48)$.

\section{Conclusions}

We show here that allergen-induced airway inflammation is dependent on IRAK-M signaling, and this is consistent with the previous reports by us and others showing the role of IRAK-M in modulating allergic airway inflammation in experimental animal models exhibiting a biphasic effect $(10,18,19)$. Our observations also indicate the differences in molecular mechanisms for modulating the pathogenesis of OVA- and HDM-induced airway inflammation. However, our mouse studies did not show the effect of IRAK-M on the regulation of airway reactivity and airway remodeling under HDM challenge, highlighting the complexity of IRAK-M in mediating pulmonary immune response to various stimuli in allergic immune response. Modulation of innate immune molecule expressed by airway epithelium in an appropriate manner might offer a novel target for allergic airway inflammation.

\section{Acknowledgments}

The authors would like to thank Professor Nikolaos G. Frangogiannis for kindly gifting IRAK-M knockout mice. The authors thank the staff at PUMC Hospital Animal Center for caring the animals.

Funding: This work was supported by grants from Natural Sciences Foundation of China (Nos.81970025, 81470229, 81900021).

\section{Footnote}

Reporting Checklist: The authors have completed the ARRIVE Reporting Checklist. Available at http://dx.doi. org/10.21037/jtd-20-2133

Data Sharing Statement: Available at http://dx.doi. org/10.21037/jtd-20-2133
Conflicts of Interest: All authors have completed the ICMJE uniform disclosure form (available at http://dx.doi. org/10.21037/jtd-20-2133). The authors have no other conflicts of interest to declare.

Ethical Statement: The authors are accountable for all aspects of the work in ensuring that questions related to the accuracy or integrity of any part of the work are appropriately investigated and resolved. Ethics approval for this study was obtained from the ethics committee of Peking Union Medical College Hospital for animal experimentation, in compliance with national or institutional guidelines for the care and use of animals (XHDW-2015-0047).

Open Access Statement: This is an Open Access article distributed in accordance with the Creative Commons Attribution-NonCommercial-NoDerivs 4.0 International License (CC BY-NC-ND 4.0), which permits the noncommercial replication and distribution of the article with the strict proviso that no changes or edits are made and the original work is properly cited (including links to both the formal publication through the relevant DOI and the license). See: https://creativecommons.org/licenses/by-nc-nd/4.0/.

\section{References}

1. Lambrecht BN, Hammad H. Allergens and the airway epithelium response: gateway to allergic sensitization. J Allergy Clin Immunol 2014;134:499-507.

2. Lambrecht BN, Hammad H. The immunology of asthma. Nat Immunol 2015;16:45-56.

3. Wenzel SE. Emergence of Biomolecular Pathways to Define Novel Asthma Phenotypes. Type-2 Immunity and Beyond. Am J Respir Cell Mol Biol 2016;55:1-4.

4. Medzhitov R, Preston-Hurlburt P, Janeway CA Jr. A human homologue of the Drosophila Toll protein signals activation of adaptive immunity. Nature 1997;388:394-7.

5. Gregory LG, Lloyd CM. Orchestrating house dust mite-associated allergy in the lung. Trends Immunol 2011;32:402-11.

6. Hammad H, Chieppa M, Perros F, et al. House dust mite allergen induces asthma via Toll-like receptor 4 triggering of airway structural cells. Nat Med 2009;15:410-6.

7. Barry J, Loh Z, Collison A, et al. Absence of Toll-IL-1 receptor 8/single immunoglobulin IL-1 receptor-related molecule reduces house dust mite-induced allergic airway inflammation in mice. Am J Respir Cell Mol Biol 
2013;49:481-90.

8. Raby BA, Silverman EK, Lazarus R, et al. Chromosome 12q harbors multiple genetic loci related to asthma and asthma-related phenotypes. Hum Mol Genet 2003;12:1973-9.

9. Balaci L, Spada MC, Olla N, et al. IRAK-M is involved in the pathogenesis of early-onset persistent asthma. Am J Hum Genet 2007;80:1103-14.

10. Liu Y, Zhang M, Lou L, et al. IRAK-M Associates with Susceptibility to Adult-Onset Asthma and Promotes Chronic Airway Inflammation. J Immunol 2019;202:899-911.

11. Kobayashi K, Hernandez LD, Galan JE, et al. IRAK-M is a negative regulator of Toll-like receptor signaling. Cell 2002;110:191-202.

12. Wu Q, Jiang D, Smith S, et al. IL-13 dampens human airway epithelial innate immunity through induction of IL-1 receptor-associated kinase M. J Allergy Clin Immunol 2012;129:825-33.e2.

13. Deng JC, Cheng G, Newstead MW, et al. Sepsis-induced suppression of lung innate immunity is mediated by IRAK-M. J Clin Invest 2006;116:2532-42.

14. Tan Q, Majewska-Szczepanik M, Zhang X, et al. IRAK-M deficiency promotes the development of type 1 diabetes in NOD mice. Diabetes 2014;63:2761-75.

15. Ballinger $M N$, Newstead MW, Zeng X, et al. IRAK-M promotes alternative macrophage activation and fibroproliferation in bleomycin-induced lung injury. J Immunol 2015;194:1894-904.

16. Hubbard LL, Moore BB. IRAK-M regulation and function in host defense and immune homeostasis. Infect Dis Rep 2010;2:e9.

17. Wu Q, Martin RJ, Lafasto S, et al. Toll-like receptor 2 down-regulation in established mouse allergic lungs contributes to decreased mycoplasma clearance. Am J Respir Crit Care Med 2008;177:720-9.

18. Nechama M, Kwon J, Wei S, et al. The IL-33-PIN1IRAK-M axis is critical for type 2 immunity in IL-33induced allergic airway inflammation. Nat Commun 2018;9:1603

19. Zhang M, Chen W, Zhou W, et al. Critical Role of IRAK-M in Regulating Antigen-Induced Airway Inflammation. Am J Respir Cell Mol Biol 2017;57:547-59.

20. Fallon PG, Schwartz C. The high and lows of type 2 asthma and mouse models. J Allergy Clin Immunol 2020;145:496-8.

21. Eisenbarth SC. Use and limitations of alum-based models of allergy. Clin Exp Allergy 2008;38:1572-5.

22. Doras C, Petak F, Bayat S, et al. Lung responses in murine models of experimental asthma: Value of house dust mite over ovalbumin sensitization. Respir Physiol Neurobiol 2018;247:43-51.

23. Juncadella IJ, Kadl A, Sharma AK, et al. Apoptotic cell clearance by bronchial epithelial cells critically influences airway inflammation. Nature 2013;493:547-51.

24. Nabe T, Matsuya K, Akamizu K, et al. Roles of basophils and mast cells infiltrating the lung by multiple antigen challenges in asthmatic responses of mice. Br J Pharmacol 2013;169:462-76.

25. Cao R, Dong XW, Jiang JX, et al. M(3) muscarinic receptor antagonist bencycloquidium bromide attenuates allergic airway inflammation, hyperresponsiveness and remodeling in mice. Eur J Pharmacol 2011;655:83-90.

26. Choi J, Choi BK, Kim JS, et al. Picroside II Attenuates Airway Inflammation by Downregulating the Transcription Factor GATA3 and Th2-Related Cytokines in a Mouse Model of HDM-Induced Allergic Asthma. PLoS One 2016;11:e0167098.

27. Cardone M, Ikeda KN, Varano B, et al. Opposite regulatory effects of IFN-beta and IL-3 on C-type lectin receptors, antigen uptake, and phagocytosis in human macrophages. J Leukoc Biol 2014;95:161-8.

28. Nials AT, Uddin S. Mouse models of allergic asthma: acute and chronic allergen challenge. Dis Model Mech 2008;1:213-20.

29. Schatz M, Rosenwasser L. The allergic asthma phenotype. J Allergy Clin Immunol Pract 2014;2:645-8; quiz 9.

30. Fricker M, Gibson PG. Macrophage dysfunction in the pathogenesis and treatment of asthma. Eur Respir J 2017;50:1700196.

31. Fitzpatrick AM, Holguin F, Teague WG, et al. Alveolar macrophage phagocytosis is impaired in children with poorly controlled asthma. J Allergy Clin Immunol 2008;121:1372-8, 1378.e1-3.

32. Lay JC, Alexis NE, Zeman KL, et al. In vivo uptake of inhaled particles by airway phagocytes is enhanced in patients with mild asthma compared with normal volunteers. Thorax 2009;64:313-20.

33. Fahy JV. Type 2 inflammation in asthma--present in most, absent in many. Nat Rev Immunol 2015;15:57-65.

34. Parker D, Prince A. Innate immunity in the respiratory epithelium. Am J Respir Cell Mol Biol 2011;45:189-201.

35. Chen W, Saxena A, Li N, et al. Endogenous IRAK-M attenuates postinfarction remodeling through effects on macrophages and fibroblasts. Arterioscler Thromb Vasc Biol 2012;32:2598-608.

36. Seki M, Kohno S, Newstead MW, et al. Critical role 
of IL-1 receptor-associated kinase- $M$ in regulating chemokine-dependent deleterious inflammation in murine influenza pneumonia. J Immunol 2010;184:1410-8.

37. Holgate ST. Innate and adaptive immune responses in asthma. Nat Med 2012;18:673-83.

38. Song C, Luo L, Lei Z, et al. IL-17-producing alveolar macrophages mediate allergic lung inflammation related to asthma. J Immunol 2008;181:6117-24.

39. Balhara J, Gounni AS. The alveolar macrophages in asthma: a double-edged sword. Mucosal Immunol 2012;5:605-9.

40. Bang BR, Chun E, Shim EJ, et al. Alveolar macrophages modulate allergic inflammation in a murine model of asthma. Exp Mol Med 2011;43:275-80.

41. Balbo P, Silvestri M, Rossi GA, et al. Differential role of CD80 and CD86 on alveolar macrophages in the presentation of allergen to T lymphocytes in asthma. Clin Exp Allergy 2001;31:625-36.

42. Burastero SE, Magnani Z, Confetti C, et al. Increased expression of the CD80 accessory molecule by alveolar macrophages in asthmatic subjects and its functional involvement in allergen presentation to autologous TH2

Cite this article as: Zhang $\mathrm{X}$, Zhang $\mathrm{M}$, Li L, Chen W, Zhou W, Gao J. IRAK-M knockout promotes allergic airway inflammation, but not airway hyperresponsiveness, in house dust mite-induced experimental asthma model. J Thorac Dis 2021;13(3):1413-1426. doi: 10.21037/jtd-20-2133 lymphocytes. J Allergy Clin Immunol 1999;103:1136-42.

43. Alcorn JF, Rinaldi LM, Jaffe EF, et al. Transforming growth factor-beta1 suppresses airway hyperresponsiveness in allergic airway disease. Am J Respir Crit Care Med 2007;176:974-82.

44. McMillan SJ, Xanthou G, Lloyd CM. Manipulation of allergen-induced airway remodeling by treatment with anti-TGF-beta antibody: effect on the Smad signaling pathway. J Immunol 2005;174:5774-80.

45. Le AV, Cho JY, Miller M, et al. Inhibition of allergeninduced airway remodeling in Smad 3-deficient mice. J Immunol 2007;178:7310-6.

46. Fattouh R, Midence NG, Arias K, et al. Transforming growth factor-beta regulates house dust mite-induced allergic airway inflammation but not airway remodeling. Am J Respir Crit Care Med 2008;177:593-603.

47. Crimi E, Spanevello A, Neri M, et al. Dissociation between airway inflammation and airway hyperresponsiveness in allergic asthma. Am J Respir Crit Care Med 1998;157:4-9.

48. Brusasco V, Crimi E, Pellegrino R. Airway hyperresponsiveness in asthma: not just a matter of airway inflammation. Thorax 1998;53:992-8. 